\title{
Primary Lymphoma of Thyroid: A Diagnostic Dilemma
}

\author{
Rajnish Kalra ${ }^{1}$, Monika Sangwan ${ }^{1}$, Namita Bhutani ${ }^{1}$, Sunita Singh ${ }^{1}$ and Ramesh Lamba ${ }^{2}$ \\ ${ }^{1}$ Depertment of Pathology, PGIMS Rohtak, India \\ ${ }^{2}$ Depertment of Surgery, PGIMS Rohtak, India
}

Submission: February 07, 2017; Published: February 14, 2017

*Corresponding author: Namita Bhutani, Department of Pathology, PGIMS Rohtak, Kailash Hills, New Delhi, India, (110065),

Tel: +91-9873101393; Fax: +91-1262-211308; Email: namitabhutani89@gmail.com; rkalra@gmail.com; monika sangwan@gmail.com; sunita6@ gmail.com; drramesh@gmail.com

\begin{abstract}
Primary Lymphoma of Thyroid is a rarely encountered clinical entity that occurs in late age intrinsically associated with Hashimotos thyroiditis, comprising of 0.6 to 5 per cent of thyroid cancers in most series. We present a case of B-cell origin thyroid lymphoma. The diagnosis was made by combined histology and immunochemistry. A 60-year-old woman presented with an enlarging neck mass with odynophagia. On admission, the sonogram of the thyroid gland showed an enlarged mass and CT scan demonstrated diffuse enlargement of the thyroid. The histological investigation revealed the presence of a diffuse large B-cell non-Hodgkin's lymphoma. The patient underwent chemotherapy. Clinicians should include primary thyroid lymphoma in the differential diagnosis of a rapidly enlarging thyroid mass. Thyroid ultrasound and fine needle aspiration cytology, using flow cytometry and Immunohistochemistry, remain the main modalities used to confirm the presence of lymphoma. The prognosis is generally excellent but can be varied because of the heterogeneous nature of thyroid lymphomas. Despite its rarity, PTL should be promptly recognized because its management is quite different from the treatment of other neoplasms of the thyroid gland.
\end{abstract}

Keywords: Primary Thyroid Lymphoma; Lymphocytic thyroiditis; Non-Hodgkin Lymphoma Thyroid Cancer

Abbreviations: PTL: Primary Thyroid Lymphoma; MALT: Mucosa-Associated Lymphoid Tissue; HT: Hashimoto's Thyroiditis; DLBCL: Diffuse Large B-Cell Lymphoma; IHC: Immunohistochemistry; RCHOP: Rituximab-Cyclophosphamide-Doxorubicine-Vincristine-Prednisone; FDGPET: Fluorine-18-Fluorodeoxyglucose Positron Emission Tomography; FNAC: Fine Needle Aspiration Cytology

\section{Introduction}

Primary thyroid lymphoma (PTL) is a rare disease that continues to produce diagnostic and therapeutic dilemmas. PTL are very rare and account for only $5 \%$ of all thyroid malignancies and approximately $3 \%$ of all non-Hodgkin's lymphoma. The annual incidence of PTL is one or two cases per million [1]. It is more prevalent in females, in the sixth to seventh decade of life with female to male ratio of 3:1 [2].

Most thyroid lymphomas are of B-cell origin. There appear to be two distinct clinical and prognostic groups of these rare tumors. The more common subtype, comprising of up to $70 \%$ of cases, is a diffuse large B-cell lymphoma. This subtype appears to have the most aggressive clinical course with almost $60 \%$ of these tumors diagnosed with disseminated disease. The other subtype is mucosa-associated lymphoid tissue (MALT) lymphomas comprising of approximately $6 \%$ to $27 \%$ of thyroid lymphomas. These have a relatively indolent course [3].

The most common presentation of thyroid lymphoma is a rapidly enlarging, painless goiter. Other symptoms such as dyspnea, dysphasia, and hoarseness may arise as a result of the pressure effects of the mass. Rarely, stridor or superior vena cava obstruction can occur. Cervical lymphadenopathy is present in the majority of cases [4]. Classic B-type symptoms such as weight loss and night sweats occur less commonly and have been reported in approximately $20 \%$ of patients. The majority of patients (30\%-60\%) are biochemically euthyroid at presentation [5].

The underlying pathogenesis of PTL remains obscure. The major risk factor for PTL is the presence of Hashimoto's thyroiditis (HT). Interestingly, although the incidence of HT in patients with PTL approaches $80 \%$, only $0.6 \%$ with HT will go on to develop PTL [6]. The association is postulated to result from the development of intrathyroidal lymphoid tissue in HT. It has also been postulated that the stimulation of antigens that are specific to the thyroid microenvironment are necessary for the development of PTL [7]. This theory is supported by the fact that more than half of thyroid lymphoma patients have a previous 
or concurrent diagnosis of chronic lymphocytic thyroiditis, suggesting that chronic antigenic stimulation may play a role in pathogenesis [8].

The overall prognosis of thyroid lymphoma has been described by the British Thyroid Association guidelines as "generally excellent"; however, prognosis is subtype dependent, and 5-year survival rates can be as low at 45\% [9]. The management and prognosis of PTL has changed with the advent of multimodal adjuvant therapy and increasing interest in and research into targeted therapies. Here, we report a case of diffuse large B-cell lymphoma (DLBCL) manifesting as a primary disorder of the thyroid gland. The importance of recognizing primary thyroid lymphoma lies in the fact that this disease is quite curable without the need for extensive surgery if recognized early and treated appropriately.

\section{Case Report}

A 60 years old woman presented to surgery outpatient department with a rapidly growing (2 weeks) thyroid enlargement along with mild odynophagia. The patient had no history of fever, night sweats or weight loss. She was a non-smoker and had no previous neck radiation exposure. The remaining medical and family history was unremarkable. Hormonal evaluation revealed increased thyrotrophic levels [TSH 32.32IU/ml, NR 0.3-4.5], normal thyroxine [123 $\mu \mathrm{g} / \mathrm{dl}$, NR 66-181] and normal tri-iodothyronine levels [1.81 nmol/L, NR 1.1-3.1]. The antithyroglobulin antibodies were markedly increased (anti-Tg $1300 \mathrm{IU} / \mathrm{ml}, \mathrm{NR}<60$ ). The initial sonogram indicated enlargement of bilateral lobes with altered echo texture and micro calcification. Bilateral cervical lymph nodes were mildly enlarged. Patient was advised FNAC thyroid swelling which revealed degenerated and intact follicular cells and large number of lymphoid cells with prominent immature component (Figure 1). Diagnosis of florid lymphocytic thyroiditis with suspected lymphomatous transformation was rendered. Patient was given steroids and L-thyroxine, $50 \mu \mathrm{g}$ replacement therapy daily. She responded well initially. But after 3 months, she came back with history of fever, weight loss, malaise and bilateral cervical swellings.

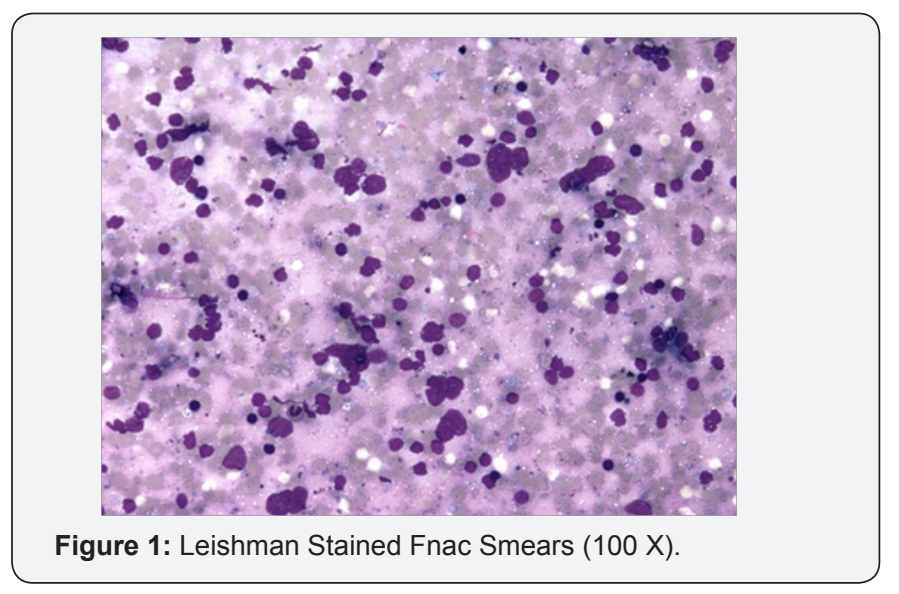

The patient underwent imaging of the neck for further evaluation.CECT Cervical region demonstrated diffusely enlarged thyroid, isotense on noncontract and showed enhancement on contrast scan. Multiple enlarged lymph nodes bilateral cervical and axillary region were also noted (Figure 2). CECT abdomen and thorax revealed abdominal, mediastinal, cervical, axillary and inguinal lymphadenopathy along with hepatosplenomegaly possibility of Koch's or Lymphoma were suggested (Figure 3).

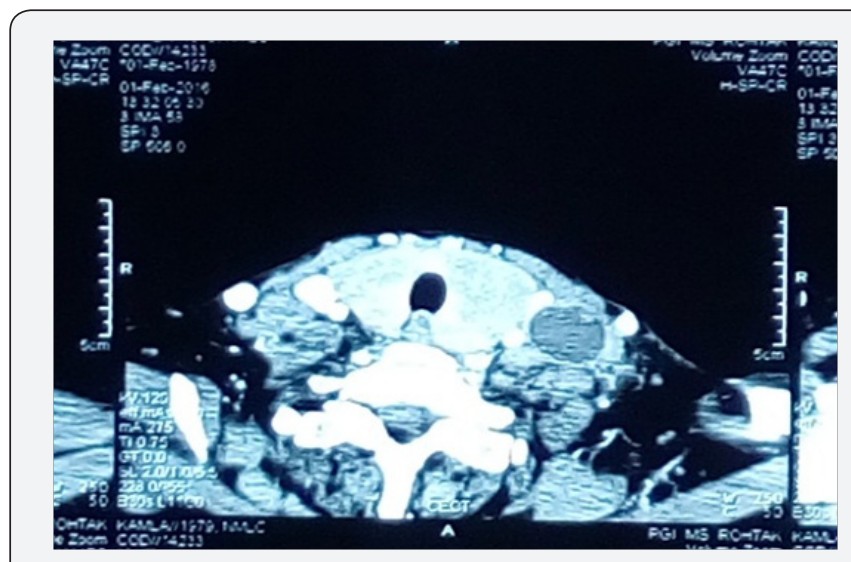

Figure 2: Axial Section Showing Diffuse Contrast Enhancement Of Thyroid With Necrotic Enlarged Lymph Node.

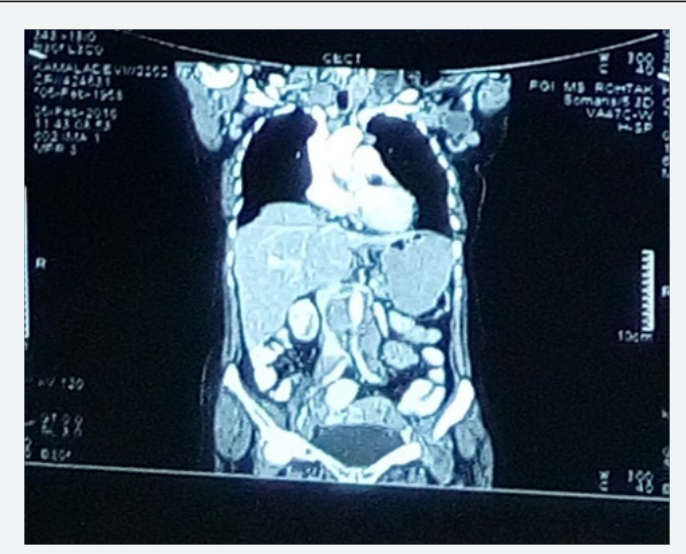

Figure 3: Coronal Section Showing Hepatomegaly with Abdominal Lymphadenopathy and Contrast Uptake.

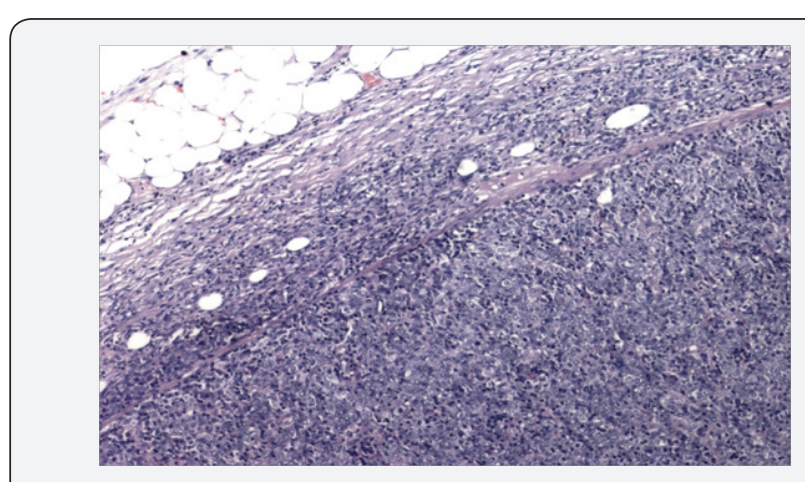

Figure 4A: Cervical Lymph Node Biopsy (H \& E- 100 X). 
Cervical lymph node biopsy was received three months after initial FNAC. Biopsy showed Non Hodgkin's Lymphoma; Diffuse Large B-Cell Lymphoma with marked necrosis and extra nodal extension (Figure $4 \mathrm{~A} \& \mathrm{~B}$ ). On Immunohistochemistry (IHC) lymphoma cells were: CD20 and CD10 positive (Figure 5A \& 5B). According to Ann Arbor guidelines for Primary Thyroid Lymphoma the patient now had stage IV E disease. Patient underwent 6 cycles of chemotherapy with RCHOP (RituximabCyclophosphamide-doxorubicine-vincristine-prednisone). She is now on regular follow up and is doing well.
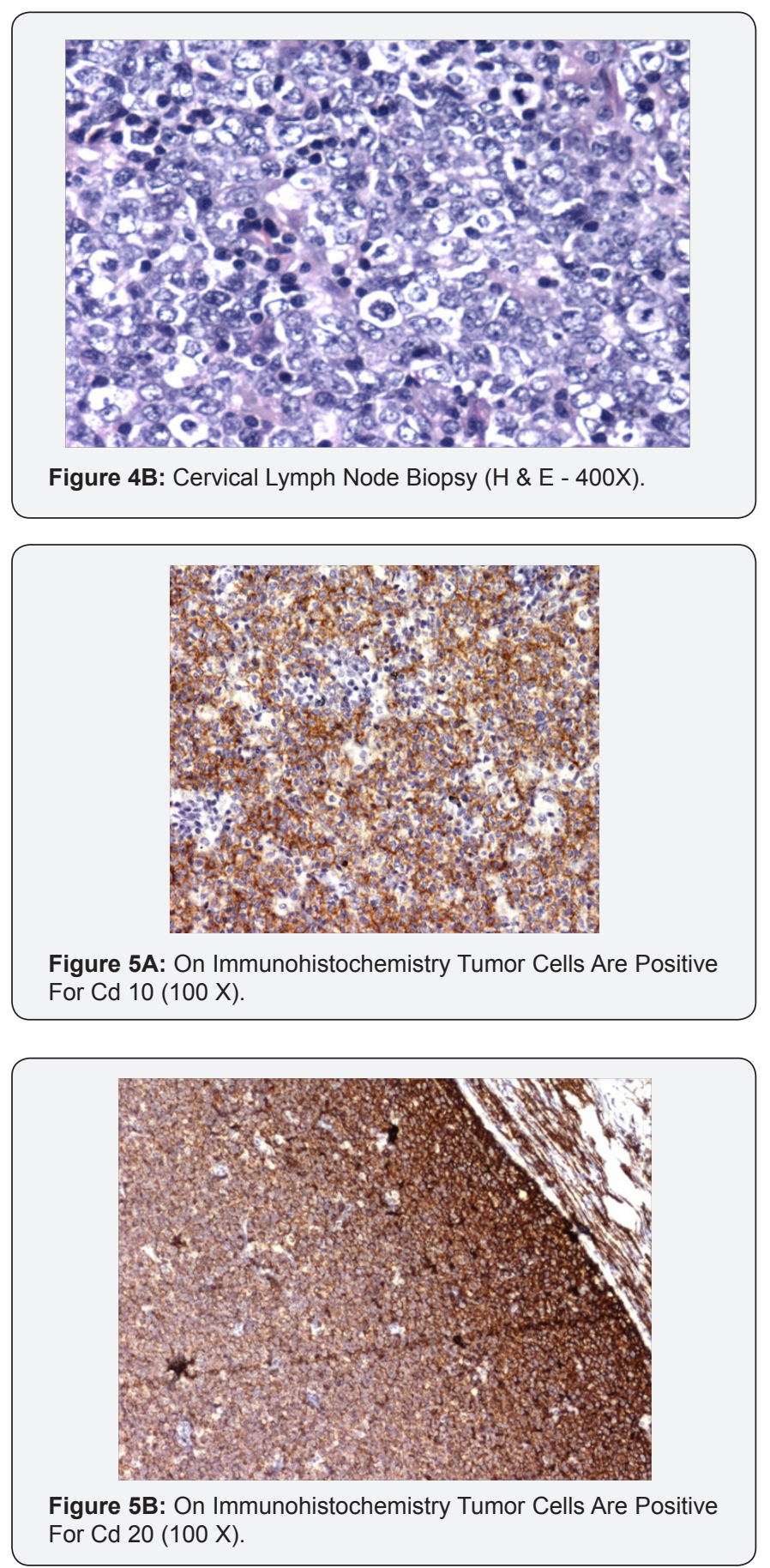

\section{Discussion}

Primary Thyroid Lymphoma (PTL) is a lymphomatous process involving the thyroid gland without contiguous spread or distant metastases from other areas of involvement at diagnosis. It is a rare tumor constituting about $1-5 \%$ of all thyroid malignancies and $1-2 \%$ of all extra nodal lymphomas, with an annual incidence of two per million [1]. Most patients present in the 7th decade of life (average age 67 years) [2]. Preexisting chronic autoimmune (Hashimoto's) thyroiditis is a wellrecognized risk factor predisposing to the development of PTL. The risk of PTL among patients with autoimmune thyroiditis is 40 times greater as compared to that of the general population. It takes a long time (20-30 years) to develop PTL after the onset of lymphocytic thyroiditis [6]. Normally, the thyroid gland does not contain native lymphoid tissue. Intra-thyroid lymphoid tissue develops under pathological conditions, and mainly in patients with autoimmune thyroiditis, probably as a result of chronic antigenic stimulation. PTLs are classified based on pathological subtypes, with each carrying a different prognosis. The two most common subtypes are diffuse large B-cell lymphoma (DLBCL) and MALT lymphoma. DLBCL accounts for up to $70 \%$ of all PTLs [3].

DLBCLs are typically positive for MS4A1 (CD20), with 75\% also positive for the BCL6 ontogeny and up to $50 \%$ positive for the BCL2. DLBCL itself has now been divided into two major cell-of-origin phenotypes with differing prognoses: a favourable germinal center B-cell-like lymphoma and a more aggressive activated B-cell like subgroup with over expression of the activated B-cell immunophenotype markers IRF4 (MUM1) and FOXP1 [10]. This group of patients, those who over expressed IRF4 (MUM1) are associated with high expression of NM23-H1 had a poorer prognosis. MALT lymphoma of the thyroid follows a relatively benign indolent clinical course and thus is more likely to present at an earlier stage and, historically, demonstrate a better response to treatment [3].

A rapidly growing (usually within 1-3 months), painless thyroid enlargement, either in the form of goiter or discrete nodule, is the most common clinical presentation in PTL. Pressure symptoms are often present. This clinical presentation can be confused with a plastic thyroid carcinoma. Because of the frequent coexistence of Hashimoto's thyroiditis, many patients are hypothyroid or under thyroid hormone replacement therapy and circulating antibodies to thyroid peroxidise are positive in these patients (60\%) [6]. Classic B-type symptoms of fever, night sweats and weight loss are less common.

Ultrasonography is the imaging modality of choice and can typically show one of three patterns: nodular, diffuse, or mixed. When presenting as a solitary mass, the radiological appearance can resemble that of anapaestic thyroid carcinoma but can be distinguished by its homogenous appearance as well as the 
lack of calcification, necrosis, and cystic degeneration within the nodule. When diffuse lymphoma is identified, it appears as a heterogeneous hypoechoic parenchyma with the presence of structures resembling septae. Magnetic resonance imaging may be more sensitive than computed tomography in the detection of extrathyroidal involvement. Fluorine-18-fluorodeoxyglucose positron emission tomography (FDG-PET) can be useful in staging and restaging or in assessing response to treatment in PTL [11]. Once the diagnosis of PTL has been established, imaging of the entire body is necessary in order to stage the patient accurately. The Ann Arbor criteria, similar to staging of lymphoma at other sites, are used for the staging of PTL.

Fine needle aspiration cytology (FNAC) is the initial technique of choice for pathological assessment of a thyroid lesion. It may present diagnostic challenges for the pathologist. Low grade lymphomas can be mistaken for chronic thyroiditis. PTL is usually of large cell type and a diagnosis of large cell lymphoma is easy on FNA and features like lack of cellular cohesion and presence of lymphoglandular bodies in the background are features strongly against a diagnosis of annalistic carcinoma. Immunocytochemistry confirms the lymphoid origin of the cells and their B or T- lineage. By contrast, cytological diagnosis of MALT- lymphomas is difficult, because of heterogeneous appearance of the neoplastic infiltrate. Distinguishing severe chronic thyroiditis from lymphoma puts us as pathologists in great difficulty when the inflammatory exudates is so expressed that it effaces the normal thyroid architecture. Moreover, such thyroiditis leads to cellular aggregates that could be classified as lymphoepitelial lesions. Morphologically, in chronic lymphocytic thyroiditis, the lymphocytes are a mixture of B and T cells but mostly T-cells. Scattered larger lymphoblasts, immunoblasts and plasma cells are also present in cases with chronic lymphocytic thyroiditis. Germinal centers are often prominent [12]. The gold standard for histologic diagnosis is considered the core needle biopsy or surgical biopsy to ensure that aggressive histologies are not missed. It yields more tissue than FNAC and maintains the architecture of the tissue and can facilitate the distinction among HT, PTL, and anapaestic carcinoma; which is not always possible with FNAC.

The gold standard for management of DLBCL is multimodal because of the typically aggressive clinical course. Surgical intervention may be required for palliation in the setting of critical airway obstruction. Intervention in this setting carries high morbidity and should be approached with caution [13]. The Surgery alone has been proposed for the management of localized intra-thyroidal lymphoma. Chemotherapy can control distant dissemination of the disease, while radiation therapy can achieve local control of the lymphoma. Most commonly, radiation therapy is used after 3-6 courses of chemotherapy. Rituximab has recently been effectively used (with cyclophosphamide, mitoxantrone, vincristine, and prednisolone) in elderly patients with diffuse large B-cell lymphoma of thyroid [14].
The identification of mutations and upregulation of cellsignaling pathways has revolutionized cancer treatment in recent years. Although molecular testing for mutations in the BRAF proto-oncogene is now used routinely to aid diagnosis and guide treatment of papillary thyroid carcinoma. Protein kinase inhibition has not yet been applied in the management of thyroid lymphoma. These findings suggest that it may be a potential therapeutic target for treatment [15].

The prognosis of DLBCL can be estimated by using the International Prognostic Index, which uses only clinical parameters. There are two indexes: one for all patients, called the "international index," and one that is age adjusted, called the "age-adjusted international index." The international index score is based on age, tumor stage, serum lactate dehydrogenase concentration, performance status, and number of extra nodal disease sites. The age-adjusted international index score is based on tumor stage, lactate dehydrogenase level, and performance status. These indexes stratify patients into four risk groups with specific 5-year survival rates, which, when compared with the Ann Arbor staging system, appear to be more accurate in predicting survival [15].

\section{Conclusion}

We describe a very rare case of primary thyroid lymphoma. Our report emphasizes the need for clinical awareness in such perplexing cases which clearly require a multidisciplinary approach for early diagnosis of such lesions and preventing delay and unnecessary surgery. Diagnosis is often difficult but clinical and radiological suspicion along with pathological features should be taken in account to reach correct diagnosis.

\section{References}

1. Ansell SM, Grant CS, Habermann TM (1999) Primary thyroid lymphoma. Semin Oncol 26(3): 316-323.

2. Graff Baker A, Roman SA, Thomas DC, Udelsman R, Sosa JA (2009) Prognosis of primary thyroid lymphoma: demographic, clinical, and pathologic predictors of survival in 1,408 cases. Surgery 146(6): 11051115.

3. Widder S, Pasieka JL (2004) Primary thyroid lymphoma. Curr Treat Options Oncol 5(4): 307-314.

4. Ruggiero FP, Frauenhoffer E, Stack BC Jr (2005) Thyroid lymphoma: A single institution's experience. Otolaryngol Head Neck Surg 133(6): 888-896.

5. Katna R, Shet T, Sengar M, Hari Menon, Siddharth Laskar, et al. (2013) Clinicopathologic studyandoutcomeanalysis of thyroidlymphomas Experiencefromatertiary cancer center. Head Neck 35(2):165-171.

6. Watanabe N, Noh JY, Narimatsu H, Takeuchi K, Yamaguchi T, et al. (2011) Clinicopathological features of 171 cases of primary thyroid lymphoma: A long-term study involving 24553 patients with Hashimoto's disease. Br J Haematol 153(2): 236-243.

7. Rossi D (2009) Thyroid lymphoma: Beyond antigen stimulation. Leuk Res 33(5): 607-609.

8. Tsang RW, Gospodarowicz MK, Sutcliffe SB, Sturgeon JF, Panzarella T, et al. (1993) Non-Hodgkin's lymphoma of the thyroid gland: Prognostic 
factors and treatment outcome. The Princess Margaret Hospital Lymphoma Group. Int J Radiat Oncol Biol Phys 27(3): 599-604.

9. Alzouebi M, Goepel JR, Horsman JM, Hancock BW (2012) Primary thyroid lymphoma: The 40 year experience of a UK lymphoma treatment centre. Int J Oncol 40(6): 2075-2080.

10. Niitsu N, Okamoto M, Nakamura N, Nakamine H, Bessho M, et al. (2007) Clinicopathologic correlations of stage IE/IIE primary thyroid diffuse large B-cell lymphoma. Ann Oncol 18(7): 1203-1208.

11. Aiken AH (2012) Imaging of thyroid cancer. Semin Ultrasound CTMR 33(2): 138-149.

12. Sangalli G, Serio G, Zampatli C, Lomuscio G, Columbo L (2001) Fine needle aspiration cytology of primary lymphoma of the thyroid: a report of 17 cases. Cytopathology 12(4): 257-263.

13. Horning SJ, Weller E, Kim K, Earle JD, O Connell MJ, et al. (2004) Chemotherapy with or without radiotherapy in limited-stage diffuse aggressive non-Hodgkin's lymphoma: Eastern Cooperative Oncology Group study 1484. J Clin Oncol 22(15): 3032-3038.

14. Mack LA, Pasieka JL (2007) An evidence-based approach to the treatment of thyroid lymphoma. World J Surg 31(5): 978-986.

15. Aggarwal N, Swerdlow SH, Kelly LM, Ogilvie JB, Nikiforova MN, et al. (2012) Thyroid carcinoma-associated genetic mutations also occur in thyroid lymphomas. Mod Pathol 25(9): 1203-1211.
Your next submission with Juniper Publishers will reach you the below assets

- Quality Editorial service

- Swift Peer Review

- Reprints availability

- E-prints Service

- Manuscript Podcast for convenient understanding

- Global attainment for your research

- Manuscript accessibility in different formats ( Pdf, E-pub, Full Text, Audio)

- Unceasing customer service

Track the below URL for one-step submission https://juniperpublishers.com/online-submission.php 